\title{
OPEN Two-dimensional arrays of vertically packed spin-valves with picoTesla sensitivity at room temperature
}

\author{
Marilia Silva ${ }^{1,2 \bowtie}$, Fernando Franco $0^{1,2,4}$, Diana C. Leitao $0^{1,2}$, Susana Cardoso $0^{1,2} \&$ \\ Paulo P. Freitas ${ }^{1,3}$
}

A new device architecture using giant magnetoresistive sensors demonstrates the capability to detect very low magnetic fields on the $\mathrm{pT}$ range. A combination of vertically packed spin-valve sensors with two-dimensional in-plane arrays, connected in series and in parallel, delivers a final detection level of $360 \mathrm{pT} / \sqrt{\mathrm{Hz}}$ at $10 \mathrm{~Hz}$ at room temperature. The device design is supported by an analytical model developed for a vertically packed spin-valve system, which takes into account all magnetic couplings present. Optimization concerning the spacer thickness and sensor physical dimensions depending on the number of pilled up spin-valves is necessary. To push the limits of detection, arrays of a large number of sensing elements (up to 440,000 ) are patterned with a geometry that improves sensitivity and in a configuration that reduces the resistance, leading to a lower noise level. The final device performance with $\mathrm{pT}$ detectivity is demonstrated in an un-shielded environment suitable for detection of bio-signals.

Emerging biomedical applications using portable sensor devices offer patients individualized and improved health monitoring ${ }^{1,2}$. High precision instruments for magnetic imaging ${ }^{3-5}$ are contactless and can offer superior outputs than electrical measurements, since magnetic signals are not attenuated by human tissue ${ }^{6,7}$. Current technologies for ultra-low magnetic field detection include Superconducting QUantum Interference Devices (SQUID $^{8}$ and Optical Pumping Magnetometry $(\mathrm{OPM})^{9,10}$ reaching femto-tesla detection levels and more recently Spin Exchange Relaxation Free (SERF) atomic magnetometers on pico-tesla range ${ }^{11,12}$. Despite the very low field detection capability, SQUIDs require cryogenic environments ${ }^{13}$ and OPMs rely on complex optical parts $^{14}$, hampering a seamless compact and wearable design. Therefore, the next generation of medical devices demands small and robust solutions with low power consumption and high sensitivity ${ }^{15,16}$, compatible with electronic integration ${ }^{17,18}$ and flexible substrates ${ }^{19}$ for enhanced portability and compactness. A reliable solution for compact devices working at a room temperature can be found in magnetoresistive (MR) sensors ${ }^{20-22}$. Several applications of giant-MR based spin-valve sensors show nT detection levels and high spatial resolution ${ }^{7,23-25}$. To push the detectivity further into $\mathrm{pT}$ range, thus becoming competitive with magnetic tunnel junctions ${ }^{26}$ or hybrid magnetic-MEMS devices ${ }^{27}$, different solutions were introduced. Large area arrays ${ }^{28,29}$ and their combination with magnetic flux guides ${ }^{26}$ have successfully delivered improved performances, at the expense of lower spatial resolution. Given the maturity of stack engineering for spin-valve multilayered thin films ${ }^{24,30}$, new design strategies are necessary to meet the demands in detection limits. We introduced the concept of a vertically packed arrangement of spin-valve sensors wherein the sensing elements are connected in parallel as seen in Fig. $1 \mathrm{a}^{31}$. The overall device resistance was reduced and consequently the noise level. An improved detectivity was demonstrated while maintaining a compact design for better spatial resolution. However, a strong decrease in sensor sensitivity is also observed due to magnetostatic coupling fields acting on the sensing layers ${ }^{32,33}$.

In this work, we present a new solution for an extremely sensitive magnetic device working at room temperature. The sensor architecture combines vertically packed spin-valves separated by an oxide spacer, with an in-plane two-dimensional array of elements (Fig. 1b,c). A new numerical model for packed spin-valves is introduced, accounting for all intra and interlayer magnetic couplings ${ }^{34}$ to support the design of the multilayered

${ }^{1}$ Instituto de Engenharia de Sistemas e Computadores - Microsistemas e Nanotecnologias (INESC-MN), 1000-029 Lisbon, Portugal. 'Instituto Superior Tecnico (IST), Universidade de Lisboa, 1040-001 Lisbon,

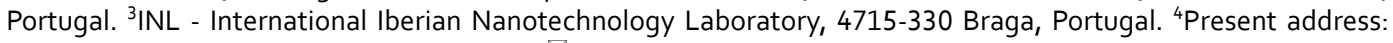
Analog Devices, Limerick V94 RT99, Ireland. ${ }^{\circledR}$ email: msilva@inesc-mn.pt 
(a) Multilayered stack

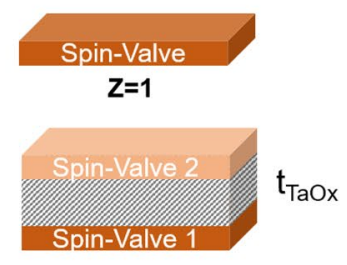

$\mathrm{Z}=2$

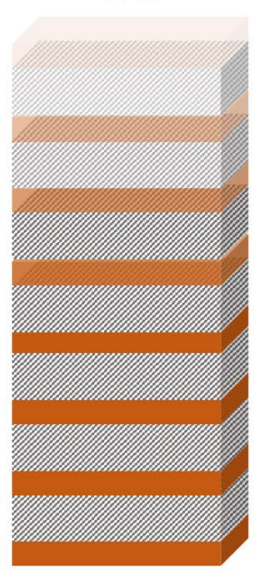

$Z=N$ (b) Planar vs packed sensors

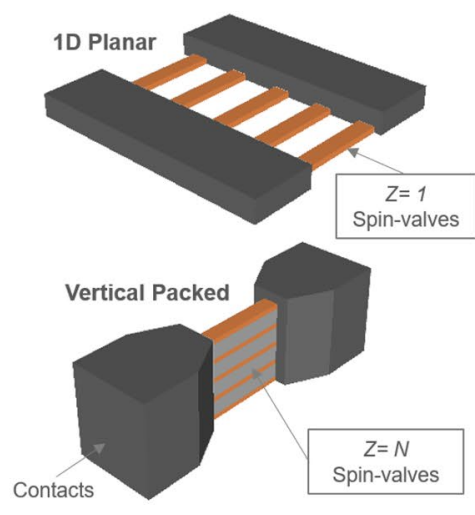

(d)

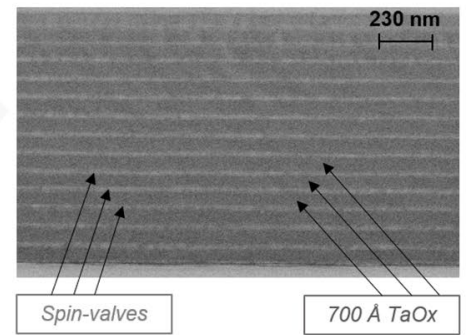

(c) Vertically packed and 2D planar array

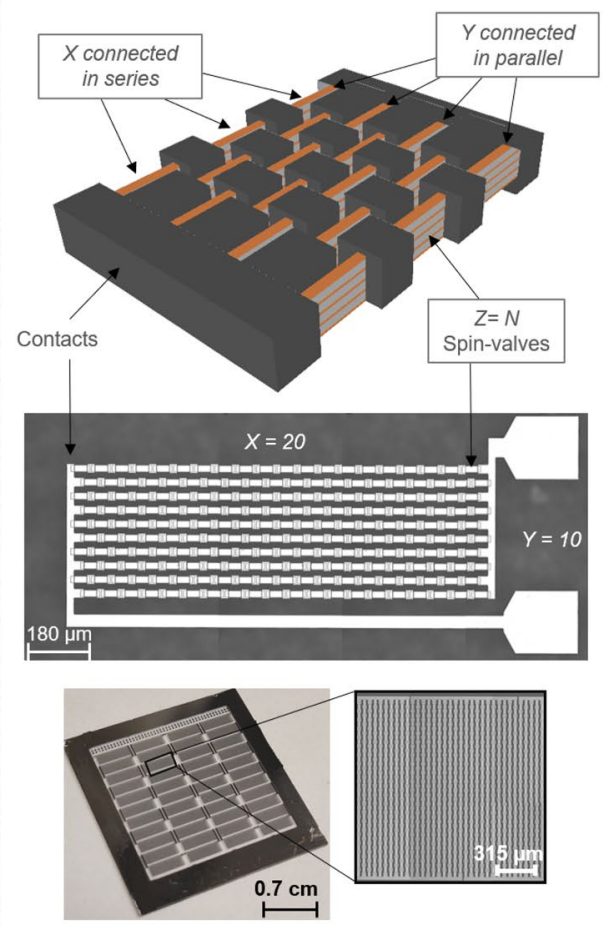

Figure 1. Designs of the spin-valve arrangements considered in this study. (a) Scheme of vertically packed multilayered thin-films with $Z=1$ for single spin-valve, $Z=2$ for two vertically packed spin-valves with different TaOx spacer thicknesses (from 500 to $1100 \AA$ ), and $Z=5,10, N$ for multiple vertically packed spinvalves with fixed $\mathrm{TaOx}$ thickness of $700 \AA$. (b) Scheme of one-dimensional planar array versus a vertically packed array; both have the elements connected in parallel. (c) Scheme of vertically packed $(Z)$ two-dimensional $(X Y)$ array of spin-valves; optical images of patterned device containing 1000 sensors $(X=20, Y=10, Z=5)$ and the final device with best performance showing 440,000 elements. (d) Scanning electron microscopy (SEM) cross sectional image of a $Z=N$ multilayer. The bright layers corresponds to the spin-valves while dark thick layers are the $\mathrm{TaOx}(700 \AA)$.

sensor. To achieve high field sensitivity in the vertically packed sensors, non-magnetic spacers can be used or sensor physical dimensions can be changed. The impact of each parameter on the magnetic behavior of the sensing device is addressed. The target is to minimize the effects of the magnetostatic coupling fields that directly affect the linear range of the sensors. The optimized final configuration combines sensors connected in series $(X)$ and in parallel $(Y)$, with vertical packing $(Z)$. For an array of 440,000 elements (Fig. 1c), a record detectivity of $360 \mathrm{pT} / \sqrt{\mathrm{Hz}}$ at $10 \mathrm{~Hz}$ is achieved at room temperature. In an unshielded environment, this device is capable of detecting magnetic fields above $500 \mathrm{pT}$ at low frequencies $(35 \mathrm{~Hz})$. With this vertical and planar packing strategy, we push the state-of-the-art in giant magnetoresistive sensors showing improved field detectivity comparable to that of series of magnetic tunnel junction sensors ${ }^{28}$ and becoming more competitive with fluxgate technology ${ }^{35}$ displaying pT detectivity ${ }^{36-38}$ with a few $\mathrm{cm}^{2}$ area. Still, the thin film magnetoresitive technology allows to further enhance the spatial resolution by increasing the number of sensors in vertical direction $(Z)$ while reducing the area in the plane; therefore reaching the requirements for bio signal detection at room temperature such as those needed for magnetocardiography ${ }^{39,40}$ and magnetomyography ${ }^{5}$.

\section{Results and discussion}

Figure 1 shows the fabricated device designs, using spin-valves. A spin-valve is a thin film multilayer structure composed of two ferromagnetic (FM) layers separated by a Cu spacer ${ }^{16}$. In a top-pinned configuration, the top FM layer has its magnetization direction fixed through exchange bias by an adjacent antiferromagnetic layer, while the magnetization of the bottom FM layer is free to rotate when a small magnetic field is applied, hence sensing layer. The current flows in the plane of the device (CIP) yielding a high resistance value when the magnetization of the two FM layers are antiparallel, and a low resistance when they align parallel to each other. Upon microfabrication, these multilayered systems can be engineered so that the sensing layer magnetization changes its direction through coherent spin rotation. A linear transition between the low and high resistance states is thus achieved being suitable for sensing applications. The micropatterned spin-valve is now sensitive to the strength and direction of the magnetic field (vector sensor) ${ }^{24,41-43}$. The full thin-film structure is obtained by alternating the growth of spin-valves and $\mathrm{TaOx}$, a scheme repeated several times for increased number of vertically packed structures ( $Z$; Fig.1d). Three groups of multilayered thin-films were prepared: (i) single spin-valve $(Z=1)$, (ii) 
(a)

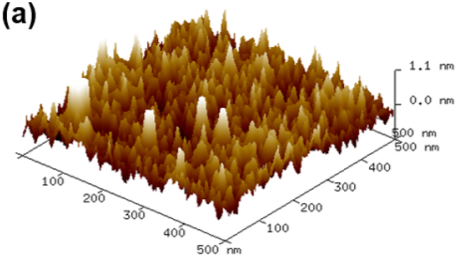

(b)

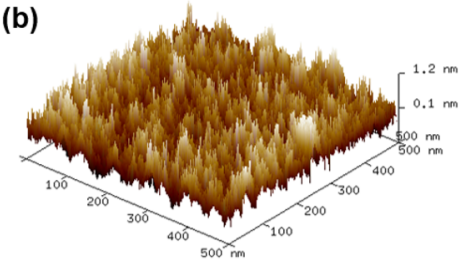

(c)

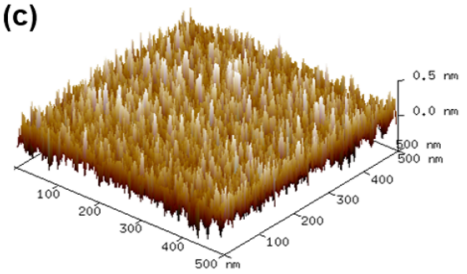

(d)

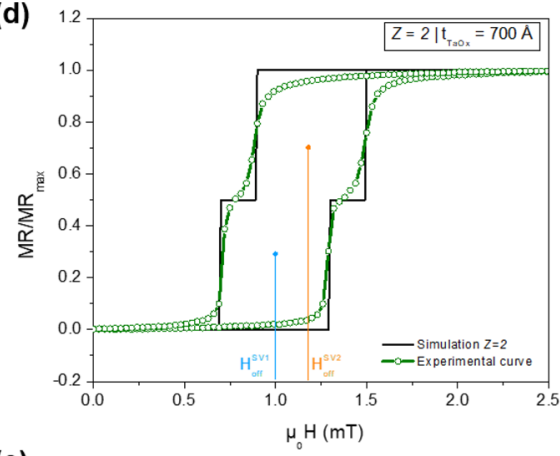

(e)

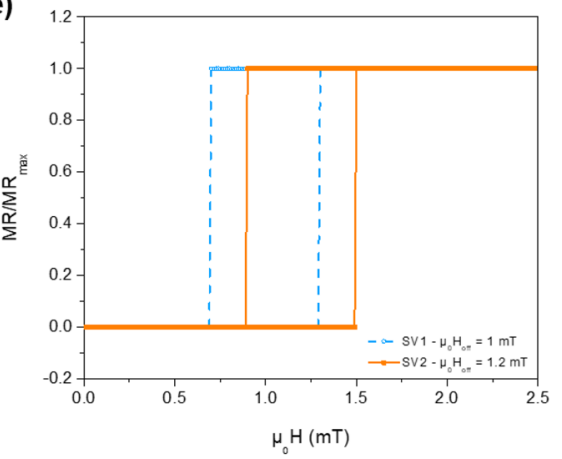

(f)

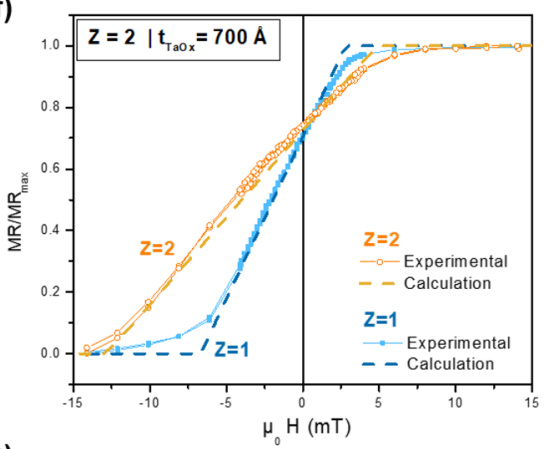

(g)

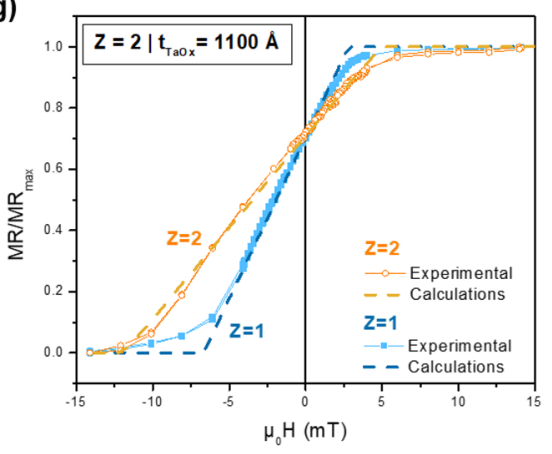

Figure 2. AFM images measured on the top surface of (a) a single spin-valve $Z=1$, (b) a spin-valve /TaOx $700 \AA$ Aspin-valve $Z=2$, and a (c) $700 \AA ̊ T a O x$ film. Transfer curves for (d, e) unpatterned samples and (f, g) micropatterned sensor. (d) Comparison for $Z=2$ of an experimental normalized $\mathrm{R}(\mathrm{H})$ transfer curve for a spacer of TaOx 700 Åand the corresponding simulated output. (e) simulated curves for each individual spinvalve in the system considering the bottom one $\left(\mathrm{SV}_{1}\right)$ with lower offset field $\left(\mathrm{H}_{\text {off }}\right)$ than the top one $\left(S V_{2}\right)$ with higher offset field. Input parameters for analytical calculations include $H_{k}$ and $M_{\text {sat }}$ values in Table 1 . Normalized transfer curves comparing the double packed spin-valves $(Z=2)$ with TaOx thickness of $(\mathbf{f})$ $700 \AA$ And (g) $1100 \AA$, and comparison with the single spin-valve structures. Experimental data for patterned structures with $w=2 \mu \mathrm{m}$ and $l=40 \mu \mathrm{m}$ is compared with the analytic calculations using Eq. (3) and input parameters of Table 1 .

two vertically packed spin-valves $(Z=2)$ with different TaOx thickness (from 500 to $1100 \AA$ ), and (iii) multiple vertically packed spin-valves $(Z=5,10)$ with fixed $\mathrm{TaOx}$ of $700 \AA$. The set with $Z=5,10$ was then patterned into $2 \mathrm{D}$ arrays (Fig. 1c) with different element size.

Unpatterned thin-film. Figure $2 \mathrm{a}-\mathrm{c}$ compares the surface topography of a single spin-valve, a spin-valve/ TaOx $700 \AA$ spin-valve structure and a $700 \AA$ TaOx film. Average roughness values of $R_{a}=2.1 \AA, 2.4 \AA$ and $1.2 \AA$ were obtained, respectively. The first value is consistent with literature for these multilayered thin-films ${ }^{44}$. The $\mathrm{TaOx}$ roughness of few $\AA$ is within optimum range to grow spin-valve structures. The $\mathrm{R}_{\mathrm{a}}$ increases slightly from $Z=1$ to $Z=2$ suggesting increased roughness in the top spin-valve structure. A higher offset field (Néel coupling) is expected ${ }^{44}$, consistent with $\mathrm{M}(\mathrm{H})$ results obtained for $Z=1$ and $Z=2$ (Fig. S1 from Supplementary Material). Figure $2 \mathrm{~d}$ shows the normalized transfer curve of the unpatterned $Z=2$ system with TaOx $700 \AA$. The minimum resistance is $\mathrm{R}_{\min }=13 \Omega$, smaller than $\mathrm{R}_{\min }=26 \Omega$ for a single spin-valve $(Z=1)$, in line with a system connected in parallel. Figure 2 e shows the theoretical output obtained for each spin-valve using the macrospin model and considering different $\mathrm{H}_{\mathrm{N}}{ }^{45}$. Equation (1) shows the total energy for the sensing layer of an unpatterned spin-valve film $(Z=1)$ :

$$
E_{\text {total }}^{\text {film }}=-\mu_{0}\left(\vec{H}_{a p p}+\vec{H}_{N}\right) \cdot \vec{M}_{S L}+K_{u} \sin ^{2} \theta .
$$

Contributions include the external applied magnetic field $\left(H_{a p p}\right)$, the Néel orange-peel coupling field $\left(H_{N}\right)$ acting on the sensing layers, and the intrinsic uniaxial magnetic anisotropy field $\left(K_{u}\right)$ defined during deposition by an applied magnetic field. $M_{S L}$ is the magnetization of the sensing layer ${ }^{45}$. For unpatterned spin-valve films the effect of the stray fields created by the pinned-layer [top $\mathrm{Co}_{80} \mathrm{Fe}_{20} 26 \AA$ ], the sensing layer $\left[\mathrm{Ni}_{80} \mathrm{Fe}_{20} 25 / \mathrm{Co}_{80} \mathrm{Fe}_{20}\right.$ $28 \AA$ ], the self-demagnetizing field due to lateral confinement, and the field created by the flowing current can be neglected. An energy minimum occurs for $\theta=\pi$ for negative external magnetic fields until $\mathrm{H}_{\mathrm{ext}}<-\mathrm{H}_{\mathrm{N}}+\mathrm{H}_{\mathrm{k}}$, and for $\theta=0$ if $\mathrm{H}_{\text {ext }}>-\mathrm{H}_{\mathrm{N}}-\mathrm{H}_{\mathrm{k}}$, yielding a squared shape (Fig. 2e) where $H_{o f f}=H_{N}$ and the coercivity $H_{c}=$ $H_{k}$. Figure $2 \mathrm{e}$ shows the calculations for $Z=2$ considering decoupled spin-valves . $\mu_{0} \mathrm{H}_{\mathrm{N}}=1 \mathrm{mT}$ is considered for the bottom spin-valve $\left(\mathrm{SV}_{1}\right)$ and $\mu_{0} \mathrm{H}_{\mathrm{N}}=1.2 \mathrm{mT}$ for the top spin-valve $\left(\mathrm{SV}_{2}\right)$. For a $Z=2$ unpatterned system the final $M R / M R_{\max }(H)$ follows the equivalent resistance formula given by: 


$$
\frac{M R}{M R_{\max }}=\frac{M_{S L} \cos \left(\theta_{1}\right)+M_{S L} \cos \left(\theta_{2}\right)}{2},
$$

where $\theta$ represents the angle between the pinned and sensing layers magnetization. These calculations correlate very well with the experimental $\mathrm{MR}(\mathrm{H})$ and justify the observed kink (Fig. 2e). The offset field values agree with the increased roughness observed by AFM.

Micropatterned sensor: impact of spacer thickness and physical dimensions. Figure $2 f, g$ compares $\mathrm{MR}(\mathrm{H})$ of micropatterned $Z=1$ and $Z=2$ spin-valve systems, with TaOx thicknesses of $700 \AA$ and 1100 $\AA$. $\mathrm{A} M R=4.5 \%$ and $\mathrm{a}_{\min }=698 \Omega$ is obtained for $Z=1$ and $\mathrm{MR}=4 \%$ and $\mathrm{R}_{\min }=397 \Omega$ for $Z=2$ (Fig. S2, Supplementary Information). $Z=1$ shows a non-hysteric behaviour with a linear range of $\mu_{0} \Delta H=9.5 \mathrm{mT}$ centered at $1.8 \mathrm{mT}$. A linear fit within $\pm 0.2 \mathrm{mT}$ around the offset field gives a sensor sensitivity $S=\frac{\Delta M R}{\Delta H}=0.4 \% /$ $\mathrm{mT}$. For $Z=2$ an increase of the linear range and the offset field is observed, when compared with $Z=1 . \Delta H$ increases by more than $80 \%$, to $17.8 \mathrm{mT}$ and $17.1 \mathrm{mT}$ for $\mathrm{TaOx}=700 \AA$ and $\mathrm{TaOx}=1100 \AA$, respectively. This corresponds to a decrease of field sensitivity of $55 \%$ and $45 \%$. The offset field also increases to $4.1 \mathrm{mT}$ for TaOx $=700 \AA$ and $3.5 \mathrm{mT}$ for $\mathrm{TaOx}=1100 \AA$.

A more complex system arises now upon micropatterning, when compared to the one described in Eq. (1). Figure 3a shows a schematic design for $Z=2$ spin-valves that includes all contributions accounted for in this new macrospin model. Magnetostatic interactions between the two spin-valves are now considered, in addition to self-demagnetizing effects, bias current fields and pinned layer stray fields. For the used $H_{a p p}$ range, the pinned layer magnetization is fixed along the magnetic easy axis (Fig. $3 a)^{45}$.

For each sensing layer $\left(\mathrm{SL}_{1}\right.$ and $\mathrm{SL}_{2}$, Fig. 3a), three inter-layer coupling terms come into play. Acting on $\mathrm{SV}_{1}$ from $S_{j}$, are (i) the dipolar field from pinned layer $\left(H_{P L}^{2-1}\right)$, (ii) the field created by the current flowing on $S V_{2}$ $\left(H_{\text {bias }}^{j-i}\right)$ and the (iii) dipolar field from sensing layer $\left(H_{S L}^{2-1}\right)$. Similar reasoning is done for the $\mathrm{SV}_{2}$ system. Due to such couplings, $\mathrm{SL}_{1,2}$ magnetization reversal in both spin-valves is not independent. Consequently, the total energy of $\mathrm{SV}_{1}\left(\mathrm{SV}_{2}\right)$ sensing layer is given by the following macrospin model:

$$
E_{\text {total }}^{S V_{1}}=-\mu_{0}\left(\vec{H}_{\text {bias }}^{1}+\vec{H}_{a p p}+\vec{H}_{N}^{1}+\vec{H}_{P L}^{1}+\frac{\vec{H}_{d e m}^{1}}{2}+\vec{H}_{\text {bias }}^{2-1}+\vec{H}_{S L}^{2-1}+\vec{H}_{P L}^{2-1}\right) \cdot \vec{M}_{S L}^{1}+K_{u} \sin ^{2}(\theta) .
$$

$H_{d e m}^{1}$ is the self-demagnetizing field while $H_{P L}^{1}$ and $H_{b i a s}^{1}$ are the stray fields originated by $S V_{1}$ pinned layer and bias current, respectively. The numerical solution of $\theta_{1}$ and $\theta_{2}$ in Eq. (3), that minimize the entire packed system energy (i.e. considering simultaneously both sensing layers) follows the algorithm depicted in Fig. 3b. The computational diagram is initialized by computing the total energy of each spin-valve. Then, the unique $\theta_{1}$ and $\theta_{2}$ that satisfy the condition of minimum energy for both spin-valves at a certain $H_{a p p}$ are found. In the end, only a single energy minimum is considered for a linear output response ${ }^{45}$.

The input values for the model are summarized in Table 1 . The $H_{N}^{1,2}, H_{k}^{1,2}$ and saturation magnetization $\left(M_{s a t}\right)$ for CoFe and NiFe were extracted from unpatterned $\mathrm{M}(\mathrm{H})$ curves. A bias current of $1 \mathrm{~mA}$ per SV is used, however only $30 \%$ is considered to flow through the Cu layer and thus contribute to $\mu_{0} H_{\text {bias }}^{1}=0.1 \mathrm{mT}$.

Figure $2 \mathrm{f}$,g compares the calculated output with the experimental curves. The calculated (normalized) $\mathrm{MR}(\mathrm{H})$ accurately depicts the experimental data obtained for micropatterned sensors. The significant increase in linear range observed for packed systems is consistent with an inter-layer coupling field that reinforces $H_{d e m}$, being the main responsible for the decrease in sensitivity. This arises from the stray fields of $S L_{1,2}$, whose orientation and intensity change according to $H_{a p p}$. Furthermore, the shift in $\mathrm{MR}(\mathrm{H})$ also indicates an additional field parallel to the sensing direction. Crossed contributions such as $H_{P L}^{1-2,2-1}$ and $H_{b i a s}^{1-2,2-1}$ reinforce the Neel coupling.

However, the packed system offset field and linear range can reach those of a single spin-valve using a first approach by increasing the spacer thickness while maintaining the high aspect ratio as shown in Fig. 3c. The calculated linear range $(\Delta H)$, dipolar field from SL (Eq. (8)) and offset field are here presented as a function of TaOx thickness. A strong $H_{S L}^{1-2,2-1}$ is obtained in the low thickness range, vanishing for $5 \mu \mathrm{m}$. From this point on, each spin-valve in the $Z=2$ system becomes magnetically independent, approaching the limit of a single spinvalve (star point in Fig. 3c). The calculated offset field is dependent on the constant values of $\mu_{0} H_{P L}^{1,2}(2.9 \mathrm{mT})$, $\mu_{0} H_{N}, \mu_{0} H_{\text {bias }}^{1,2}$ and changing values of $\mu_{0} H_{P L}^{1-2}$ or $\mu_{0} H_{P L}^{2-1}$. When the latter becomes negligible $\left(t_{T a O x}>2.5 \mu\right.$ $\mathrm{m})$, the $Z=2$ offset field is comparable to a single spin-valve.

As alternative to magnetically decouple the vertically packed spin-valves, long deposition times of a thick spacer layer can be substituted by engineered physical dimensions of the sensor $(w$ and $l)$. Using the developed macro spin model for double packed spin-valves $(Z=2)$ and considering a thin spacer layer (e.g. $700 \AA$ ), the contributions from the stray fields decrease with increasing $w$, therefore the $H_{S L}^{j-i}$ along the sensor width decreases very sharply until $w=10 \mu \mathrm{m}$ and mostly vanishes above $w=80 \mu \mathrm{m}$ (see Fig. S3 (a) in Supplementary Information). This effect consequently improves the sensitivity and reduces the offset approaching those of a single spin-valve with fixed $w=2 \mu \mathrm{m}$ (see Fig. S3 (b) in Supplementary Information). Furthermore, this strategy can be effectively employed for $Z>2$. The real impact on the MR(H) curve, of altering the physical width $(w=2$ to $100 \mu \mathrm{m})$ in packed systems $(Z=5$ and $Z=10)$, was experimentally evaluated and compared with calculated curves from the macro spin model for $Z=1$ and $Z=10$ (Fig. 4a,b). Results from macro spin model for $Z=5$ and 10 are shown in Fig. S3 (c)-(h) of the Supplementary Material. Figure 4c compares the sensitivity and hysteresis in all studied structures. Three regions are visible: (i) linear and minimal hysteresis $\left(\mu_{0} \mathrm{H}_{\mathrm{c}}<0.05 \mathrm{mT}\right)$, (ii) linear with small hysteresis $\left(\mu_{0} \mathrm{H}_{\mathrm{c}}>0.1 \mathrm{mT}\right)$, and (iii) not linear $\left(\mu_{0} \mathrm{H}_{\mathrm{c}}>0.4 \mathrm{mT}\right)$ and saturated at zero field. 
(a)

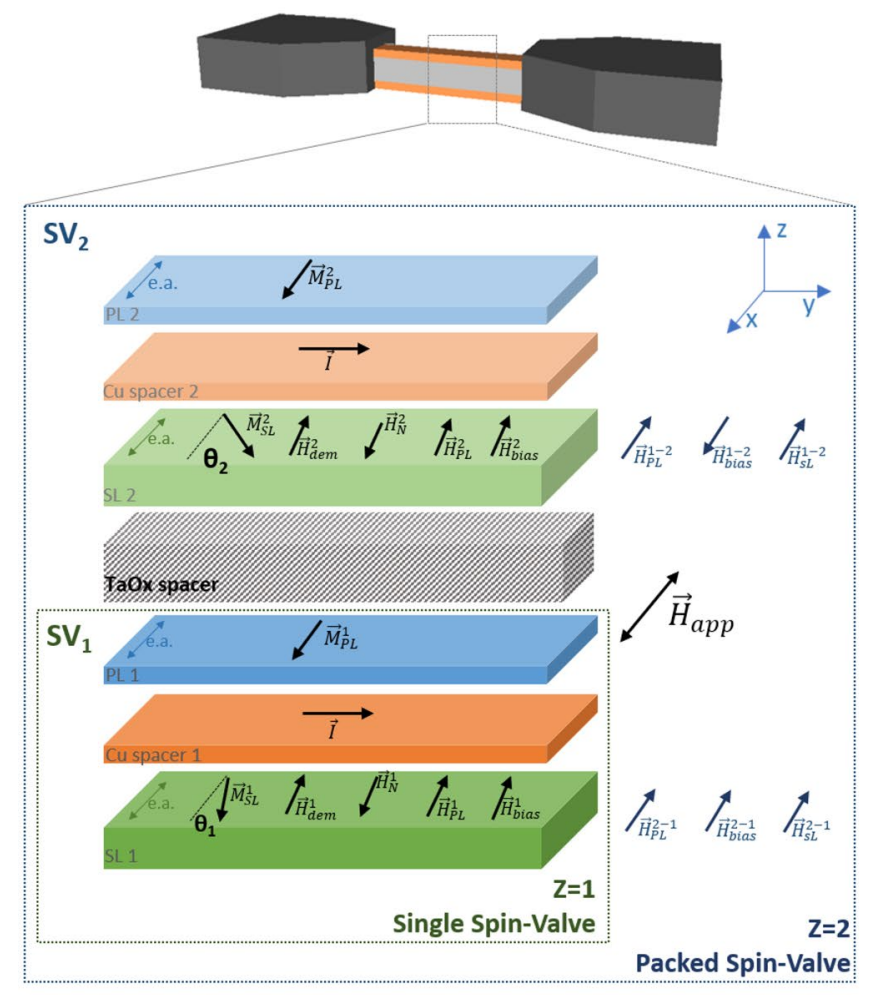

(b)

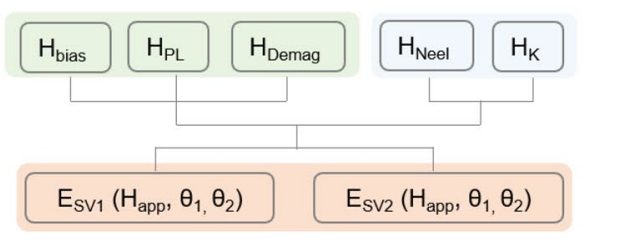

A
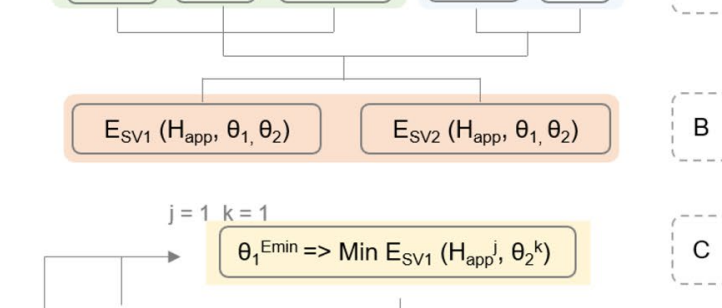

C

(-

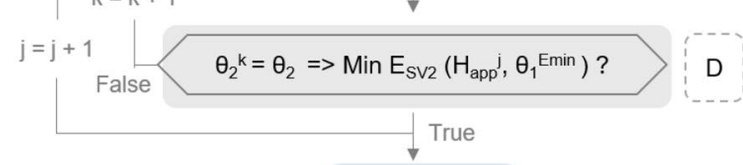

(c)

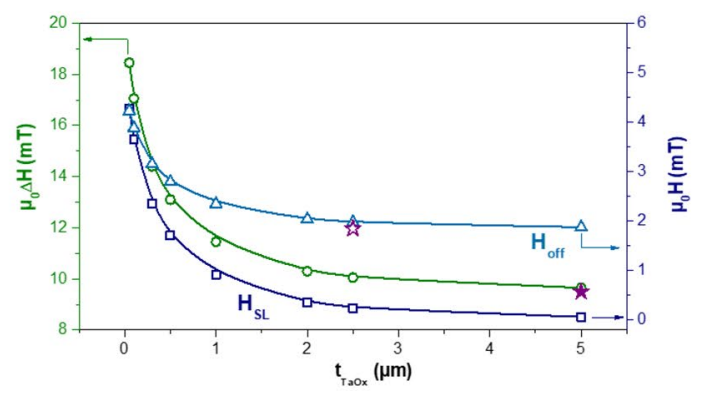

Figure 3. (a) Schematic of two spin-valves separated by a TaOx thick spacer for the implementation of the analytical model. $\mathrm{SV}_{1}$ stands for the bottom spin-valve (deposited first) and $\mathrm{SV}_{2}$ is the top spin-valve grown on top of the TaOx spacer. All magnetic field contributions acting on the sensing layers are represented $\left(H_{a p p}, H_{o f f}^{1,2}\right.$, $\left.H_{P L}^{1,2}, H_{\text {bias }}^{1,2}, H_{\text {dem }}^{1,2}\right)$. Crossed inter-layer couplings are also added, namely the dipolar fields from $\mathrm{SV}_{2}$ pinned layer and sensing layer $\left(H_{P L}^{2-1}, H_{S L}^{2-1}\right)$ acting on $\mathrm{SV}_{1}$ (and vice-versa), and field created from the flowing current $H_{\text {bias }}^{2-1}$ (and vice-versa). $M_{S L}^{1,2}$ and $M_{P L}^{1,2}$ are the magnetizations of the sensing (SL) and pinned (PL) layers, respectively. (b) Employed algorithm to solve the $Z=2$ spin-valve system. At stage A, the self-demagnetizing and stray fields acting in each SL are calculated, while offset field and induced uniaxial anisotropy are obtained from experimental $\mathrm{R}(\mathrm{H})$ curves of unpatterned samples. At stage $\mathrm{B}$ two matrices $E_{S V 1}$ and $E_{S V 2}$ are generated with the total energy of the SL of each $S_{1,2}$ as a function of external field applied $\mathrm{H}_{a p p}$ and all combinations of $\theta_{1}$ and $\theta_{2}$. At stage $\mathrm{C}$ is initialized a loop to find the $\theta$ that minimize the energy for both spin-valves at a given applied field. Firstly, $\theta_{1}$ that minimizes the $E_{S V 1}$ is found for $\mathrm{H}_{a p p}$ and $\theta_{2}^{k}$.Then, at stage $\mathrm{D}$ is verified if the conditions at $\mathrm{C}$ minimizes simultanously the $\mathrm{E}_{S V 2}$, otherwise the angle $\theta_{2}$ is swept until both energies are minimized. Consequently, the previous loop restart for a different $H_{a p p}$ in order to generate the output at stage E. (c) Calculated linear range (green; Eq. (3)), sensing layer stray field (blue; Eq. (8)) and offset field (light blue; Eq. (3)) as a function of TaOx spacer thickness for $Z=2$. The star points indicates experimental values for a single SV of $\Delta H($ closed $\star)$ and $H_{o f f}($ open $\star)$.

\begin{tabular}{|l|l|l|l|l|l|l|l|}
\hline Parameter & $w$ & $l$ & $\boldsymbol{\mu}_{0} \boldsymbol{H}_{\boldsymbol{N}}^{1}$ & $\boldsymbol{\mu}_{0} \boldsymbol{H}_{\boldsymbol{N}}^{2}$ & $\boldsymbol{\mu}_{0} \boldsymbol{H}_{\boldsymbol{k}}$ & $\boldsymbol{M}_{\text {sat }}^{\text {NiFe }}$ & $\boldsymbol{M}_{\text {sat }}^{\mathrm{CoFe}}$ \\
\hline Value & $2 \mu \mathrm{m}$ & $40 \mu \mathrm{m}$ & $1 \mathrm{mT}$ & $1.2 \mathrm{mT}$ & $0.3 \mathrm{mT}$ & $930 \mathrm{kA} / \mathrm{m}$ & $1250 \mathrm{kA} / \mathrm{m}$ \\
\hline
\end{tabular}

Table 1. Input parameters used for analytic calculations.

For $Z=1$, linear outputs are achieved up to $w=5 \mu \mathrm{m}$, becoming linear and hysteretic until $w=10 \mu \mathrm{m}$ and then completely squared. For $Z=5$ and $Z=10$, small hysteresis appears above $w=5 \mu \mathrm{m}$,loosing the linearity for $w>80 \mu \mathrm{m}$.

In region (i), the sensitivity for $Z>1$ is lower than $Z=1$ since dipolar coupling and demagnetizing field contribute to enlarge the linear operation range. In region (ii), packed spin-valves are still linear although with a small hysteresis for a broad range of $w(5 \mu \mathrm{m}<w<80 \mu \mathrm{m})$. This translates into a sensitivity similar to that observed for a linear $Z=1$ spin-valve $(0.4 \% / \mathrm{mT})$. For region (iii), $w>80 \mu \mathrm{m} \mathrm{MR}(\mathrm{H})$ becomes square, as the intrinsic anisotropy dominates, hindering its use as a magnetic field sensor. An additional magnetic field, applied perpendicular to the sensing direction, is effective in reducing this hysteresis but adds complexity to the fabrication of a compact device design (Fig. S4 of Supplementary Material) ${ }^{26}$. 

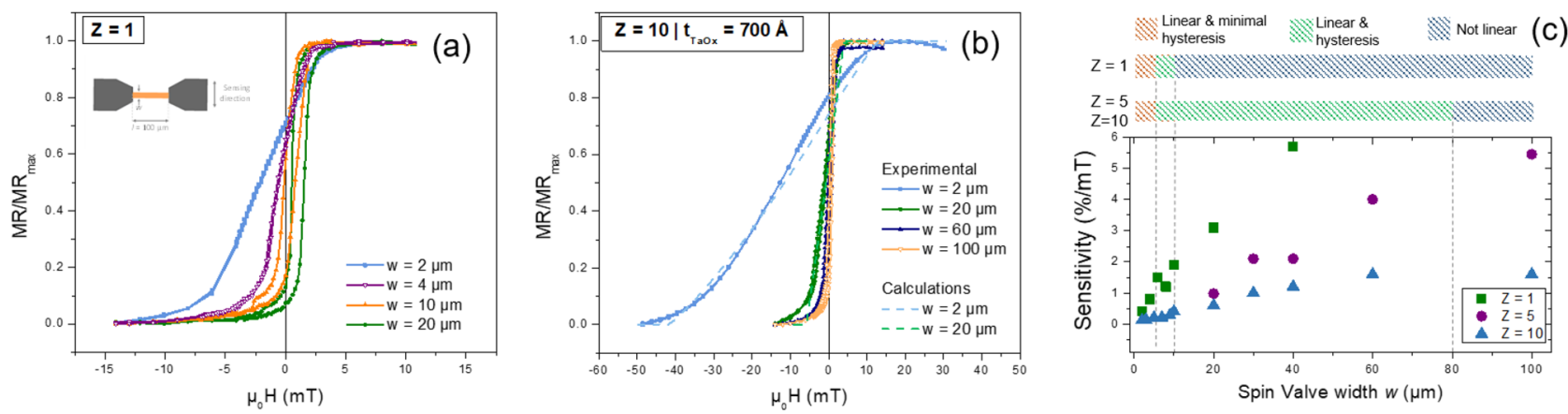

Figure 4. Normalized transfer curve of spin-valve sensor for (a) $Z=1$ and (b) $Z=10$ by varying the width from 2 to $100 \mu \mathrm{m}$. For $Z=10$ is also shown calculated curves from the developed macrospin model for $\mathrm{w}=2 \mu \mathrm{m}$ and $20 \mu \mathrm{m}$. (c) Sensitivity as a function of the sensor width obtained at the most sensitive point of the curve. Three regions are defined to describe the sensor behavior accounting for its hysteresis and linear output.
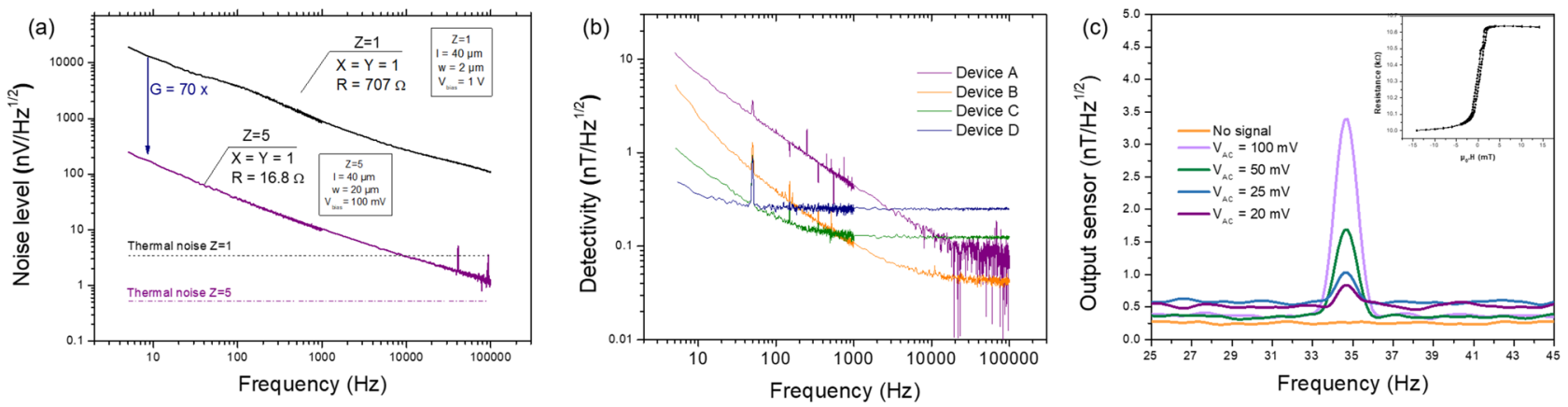

Figure 5. (a) Noise curves for a single $Z=1$ and $Z=5$ packed spin-valves, measured at the most sensitive point of the curve $\left(H_{a p p}=0\right)$. The two dashed lines indicate the calculated thermal noise baseline. The bias voltage was calculated in order to have about $1 \mathrm{~mA}$ flowing per each spin-valve. (b) Detectivity curves obtained through the ratio between noise and sensitivity for four devices (Device A, B, C, D in Table 2) with different number of sensors. Device A was biased with $1 \mathrm{~V}$ and the others with 3 V. (c) Output of Device D under a bias voltage of $3 \mathrm{~V}$, in an unshielded environment and subjected to variable voltage amplitude applied to the external coil at $35 \mathrm{~Hz}$. The inset shows $\mathrm{R}(\mathrm{H})$ of Device D with $\mathrm{MR}=6.3 \%, R_{\min }=10 \mathrm{k} \Omega, \mu_{0} H_{\text {off }}=0.2 \mathrm{mT}, \mu_{0} H_{c}=0.25$ $\mathrm{mT}$ and a zero field sensitivity of $1.7 \% / \mathrm{mT}$ ( or $17.2 \mathrm{~V} / \mathrm{V} / \mathrm{T}$ ).

2D arrays: reducing noise, increasing detectivity. In spin-valve sensors the main sources of noise are thermal and $1 / \mathrm{f} \mathrm{noise}^{29}$, being the total noise voltage power spectral density $\left(S_{V}\right)$ given by:

$$
S_{V}^{2}=4 k_{B} T R+\frac{\gamma V^{2}}{n_{c} f},
$$

where $k_{B}$ is the Boltzmann constant, $T$ the temperature, $R$ the sensor resistance $\left(R=R_{\square} \frac{l}{w}\right), \gamma$ the Hooge $1 / \mathrm{f}$ noise parameter, $V$ the bias voltage, $n_{c}$ the number of charge carriers $\left(n_{c}=V \times C=w l t C\right)$, and $f$ the frequency. When the system evolves to a packed configuration and its physical dimensions are altered, the noise is given by:

$$
S_{V}^{2}=4 k_{B} T R_{\square} \frac{l}{w Z}+\frac{\gamma V^{2}}{Z w l t C} \frac{1}{f} .
$$

where $Z$ is the number of packed sensors. By vertically stacking sensors with a large area $(w \times l)$ and connected them in parallel, a lower resistance is obtained, promoting a general noise reduction over the entire frequency spectrum. Figure 5a compares the noise level for two devices $Z=1, w=2 \mu \mathrm{m}$ and $Z=5, w=20 \mu \mathrm{m}$, chosen for their optimal linear output according to the discussion above. Measurements are performed under a bias voltage of $1 \mathrm{~V}$ and $100 \mathrm{mV}$, for $Z=1$ and 5 respectively, thus ensuring $1 \mathrm{~mA}$ per spin-valve.

Consequently, two devices $i$ and $j$ with different properties $(i) Z_{i}, V_{i}, w_{i}$ and $(j) Z_{j}, V_{j}, w_{j}$ reveal a gain $G_{j i}$ in noise level (ratio between the two $S_{V}$ ) at low frequency given by: 


\begin{tabular}{|c|c|c|c|c|}
\hline Device & A & B & C & D \\
\hline Lateral size $w(\mu \mathrm{m})$ & \multicolumn{4}{|l|}{20} \\
\hline Vertically packed $Z$ & \multicolumn{4}{|l|}{5} \\
\hline Connected in series $X$ & \multicolumn{4}{|l|}{20} \\
\hline Connected in parallel $Y$ & \multicolumn{4}{|l|}{10} \\
\hline Device A repetitions $\left(N_{A}\right)$ & 1 & 10 & 110 & 440 \\
\hline Number of sensors & 1000 & 10,000 & 110,000 & 440,000 \\
\hline Detectivity@10 Hz $(\mathrm{nT} / \sqrt{\mathrm{Hz}})$ & 6.9 & 2.4 & 0.71 & 0.36 \\
\hline Calculated gain@10 Hz & 1 & 3.2 & 10.0 & 21.0 \\
\hline Measured gain@ $10 \mathrm{~Hz}$ & 1 & 2.9 & 9.7 & 19.4 \\
\hline
\end{tabular}

Table 2. Details of the characterized vertically-packed $2 D$ arrays. $Z$ is the number of vertical spin-valves, $X$ connected in series and $Y$ in parallel. Sensor A represents an array of $X=20, Y=10$ and $Z=5$ with a total number of 1000 sensors. Sensor B, C and D are the array A replicated 10,110 and 440 times, respectively. The detectivity gains, calculated and measured, are obtained relative to Device A.

$$
G_{j i}=\sqrt{\left(\frac{V_{i}}{V_{j}}\right)^{2} \frac{w_{j}}{w_{i}} \frac{Z_{j}}{Z_{i}}} .
$$

An improvement (decrease) of 70 times in the noise level is obtained for $Z=5$ at low frequencies. Although, the spatial resolution increases by a factor of 10 , the decrease in the noise level is more significant.

Our approach goes beyond packing sensors in the vertical direction $(Z)^{31-33}$, pushing further on the detection level by using in-plane two-dimensional arrays with $X$ sensors connected in series and $Y$ sensors connected in parallel. The noise level of such $X Y Z$ array is now given by:

$$
S_{V}^{2}=4 k_{B} T \frac{X}{Y Z} R+\frac{\gamma X V^{2}}{Y Z w l t C} \frac{1}{f}
$$

An overall improvement of $\sqrt{\frac{X}{Y Z}}$ is therefore expected for the entire noise spectrum considering the same physical dimensions.

A compromise between the physical dimension and sensitivity exists in packed system, and extends to planar arrays. Comparing a device with $Z=1, X=40, Y=25(w=2 \mu \mathrm{m})$ and another with $Z=5, X=20, Y=10$ $(w=20 \mu \mathrm{m})$, a theoretical gain of $6.4 \times$ at low frequency is predicted. Experimentally, a gain of 5.6× with an improvement in spatial resolution of $1.5 \times$ is achieved. In fact, only by increasing $Z$ from 5 to 10 , while maintaining physical dimensions and $X Y$ number of sensors, a larger improvement in spatial resolution of $2 \times$ is obtained, although the expected gain in noise is only $\sqrt{2}$ (Fig. S6 in Supplementary Information).

Figure $5 \mathrm{~b}$ compares the detectivity level for different devices (A to D), consisting of $Z=5$ vertically-packed $2 \mathrm{D}$ arrays of sensors with $w=20 \mu \mathrm{m}$ (Table 2). The detectivity is the ratio of the sensor's noise level and its sensitivity (signal-to-noise ratio equal to 1). The choice of geometrical features considers a compromise between spatial resolution and detectivity. $Z=10$ could be used for lower noise, however a large $w$ had to be used to ensure linearity and higher sensitivity (see Supplementary Information).

At $10 \mathrm{~Hz}$, Device A shows a minimum detectable field of $6.9 \mathrm{nT} / \sqrt{\mathrm{Hz}}$. Since, the detectivity improves by $1 / \sqrt{X Y Z}$ (Eq. (7)), pT resolution is possible by increasing the total number of sensors by at least 100 times (gain of $10 \times$ ). Device A was therefore replicated several times (Devices B, C and D) to achieve the targeted performance (Fig. 1c; Table 2). Due to limitations of the measuring system, Device A was biased with $1 \mathrm{~V}$ and the others with $3 \mathrm{~V}$. Nevertheless, at low frequencies $(10 \mathrm{~Hz})$ the range for biomedical aplications, the detectivity does not depend on the voltage. The same is not valid for high frequencies where the detectivity varies with $1 / \sqrt{V}$. Comparing the performance at $10 \mathrm{~Hz}$, the best detectivity is obtained for Device D (440,000 sensors) with $360 \mathrm{pT} / \sqrt{\mathrm{Hz}}$ and a gain of $19.4 \times$. Table 2 summarizes the results obtained for all devices showing a good correlation between measured gain and calculated gain according to $\sqrt{N}$. The drawback of this strategy is the increase in occupied area, which can be partially compensated by larger $Z$.

Measurements in an un-shielded environment. Device $\mathrm{D}$ is then used to measured a very low magnetic field without magnetic shielding, as in a real experiment environment. Figure $5 \mathrm{c}$ shows the magnetic field detected by the device. This is obtained from converting of the voltage output into field units using the device sensitivity. The inset of Fig. $5 c$ shows the $\mathrm{R}(\mathrm{H})$ transfer curve biased with $3 \mathrm{~V}$ and exhibiting a sensitivity of 17.2 V/V/T. The baseline and limit of detection of $360 \mathrm{pT} / \sqrt{\mathrm{Hz}}$ are obtained with a measurement without any AC signal similar for shielded environment. Then a voltage sweep from 100 to $20 \mathrm{mV}$ was applied, resulting in a magnetic field of $3400 \mathrm{pT} / \sqrt{\mathrm{Hz}}$ and $800 \mathrm{pT} / \sqrt{\mathrm{Hz}}$ for the limits of applied voltage. Due to thermal drifts, an increase in the signal baseline during the measurements is visible. The last measurement shows a SNR $=1.7$ (4.6 $\mathrm{dB}$ ) setting the threshold for signal detection. 


\section{Conclusions}

By merging vertically-packed and in-plane arrays of sensors into a highly sensitive device we are able to detect picoTesla fields in an unshielded environment. Optimized packed spin-valve systems are demonstrated, overcoming the loss of sensitivity due to magnetostatic couplings. Two different routes to mitigate dipolar couplings and improve sensitivity are outlined by the implemented numerical model. This new tool encloses all crossed magnetic coupling terms and allowed us to explore their influence in the self-demagnetizing field of the sensing layers for micropatterned double-packed spin-valve architectures. The first approach requires changing the thickness of TaOx spacer while maintaining the high aspect ratio of the sensing elements, setting the threshold for complete magnetic decoupling at $2.5 \mu \mathrm{m}$ spacer thickness which poses challenges for experimental implementation. The second route relies on changing the sensor element physical dimensions (low aspect ratio) while preserving a thin and low roughness spacer. Experimentally, two-dimensional arrays of spin-valve elements with $Z$ up to 10 , enclosing a thin spacer of $700 \AA$ with low roughness and optimum geometry were successfully fabricated by using standard deposition and micropatterning techniques. An array with five vertically-packed spin-valves and 20 sensing elements connected in series and 10 in parallel replicated 440 times, shows the capacity of measuring pT signals above a detection threshold of $360 \mathrm{pT} / \sqrt{\mathrm{Hz}}$ at $10 \mathrm{~Hz}$, fitting the specification for biomedical signal detection.

\section{Methods}

Growth of vertically packed multilayers. The multilayer thin-films and the insulator spacer were grown with ion beam deposition tools (N3000/N3600). The spin-valve stack used is composed of [Ta $20 / \mathrm{Ni}_{80} \mathrm{Fe}_{20}$ $25 / \mathrm{Co}_{80} \mathrm{Fe}_{20} 28 / \mathrm{Cu} 24 / \mathrm{Co}_{80} \mathrm{Fe}_{20} 26 / \mathrm{Ir}_{24} \mathrm{Mn}_{76} 70 / \mathrm{Ta} 50(\AA)$ ]. The spacer between consecutive spin-valves is tantalum oxide (TaOx). Ta is well known to act as a good buffer for magnetosresistive stacks ${ }^{46,47}$. TaOx was grown by depositing Ta assisted by a non accelerated $\mathrm{O}_{2}$ plasma $\left(\mathrm{V}^{+}=\mathrm{V}^{-}=0 \mathrm{~V}\right.$; RF power: $160 \mathrm{~W}, \mathrm{O}_{2}$ flow: $\left.10 \mathrm{sccm}\right)$.

Sensor microfabrication. The spin-valve thin-films were patterned by photolithography and ion milling etching at 70 degrees to obtain a steep lateral profile along the full thickness. For $\mathrm{Z}=1$ and 2 and varying TaOx thickness, a length of $l=40 \mu \mathrm{m}$ and width $w=2 \mu \mathrm{m}$ was used to define the sensor. For $Z=5$ and 10 and $\mathrm{t}_{\mathrm{TaOx}}=700 \AA$, the structures were patterned with $l=40 \mu \mathrm{m}$ and $100 \mu \mathrm{m}$, changing $w$ from 20 to $100 \mu \mathrm{m}$. Planar arrays $(Z=1)$ and vertically packed arrays $(Z=5$ and 10$)$ were fabricated with the number of sensors connected in series $(X)$ changing from 10 to 40 , and connected in parallel $(Y)$ from 2 to 25 sensors (Fig. 1c). Electrical contacts were defined by liftoff of $\mathrm{Al}_{98.5} \mathrm{Si}_{1.0} \mathrm{Cu}_{0.5} 3000 / \mathrm{Ti}_{12.5} \mathrm{~W}_{50} \mathrm{~N}_{37.5} 150[\AA]$ and passivated with $\mathrm{Al}_{2} \mathrm{O}_{3} 1000 \AA$. Annealing was then performed at $250^{\circ} \mathrm{C}$ for 30 minutes, and the sample cooled down under an external magnetic field of $1 \mathrm{~T}$.

Characterizations. Unpatterned films were characterized by Vibrating Sample Magnetometry (VSM) and electrically using four point probe measurement. Scanning Electron Microscopy (SEM) was performed in a Raith 150 Ebeam/SEM system. Atomic Force Microscopy (AFM) was performed to access the impact on topography from different TaOx spacer thicknesses. Areas of $500 \mathrm{~nm}$ by $500 \mathrm{~nm}$ were scanned, with a frequency of $1 \mathrm{~Hz}$ and 256 lines.

The patterned structures were measured using a DC two-point probe method with $1 \mathrm{~mA}$ bias current within a field range of $\pm 14 \mathrm{mT}$. The sensor noise spectra were acquired within a frequency range of $\mathrm{dc}-100 \mathrm{kHz}$ at room temperature on a magnetically shielded box. The device under test is biased through a battery to mitigate the $50 \mathrm{~Hz}$ power network component while the output is amplified 100× by a low noise amplifier SIM910 that is connected to a spectrum analyzer.

The evaluation of the limits of detection in an unshielded environment used a low resistance Helmholtz coil $(\mathrm{N}=8$ and $\mathrm{r}=2.5 \mathrm{~cm})$ connected to a $10 \mathrm{k} \Omega$ resistor. The circuit is biased with a AC signal with a voltage changing from $100 \mathrm{mV}$ to $20 \mathrm{mV}$ at a frequency of $35 \mathrm{~Hz}$ to create an AC magnetic field (0.34 pT/nA). Each measurement was done with a gain of 100 through a low noise amplifier and averaged 50 times within a resolution bandwidth (RBW) of $1 \mathrm{~Hz}$ between 5 and $200 \mathrm{~Hz}$.

Calculation of the demagnetizing field. For the model considered in Eq. (3), only uniformly magnetized media are assumed, and thus a macroscopic description using magnetostatic equations is chosen to calculate $H_{\text {dem }}^{1,2}, H_{P L}^{1,2}$ and crossed terms $H_{P L}^{1-2}, H_{P L}^{2-1}$ and $H_{S L}^{1-2}, H_{S L}^{2-1}{ }_{48}$ :

$$
\vec{H}_{r}=\frac{1}{4 \pi} \int_{S} \frac{\overrightarrow{n \cdot \vec{M}\left(\overrightarrow{r^{\prime}}\right)\left(\vec{r}-\overrightarrow{r^{\prime}}\right)}}{\left|\vec{r}-\overrightarrow{r^{\prime}}\right|},
$$

where $M$ is the magnetization of the sensing or the pinned layer. Likewise, the field created by the bias current $\left(H_{\text {bias }}^{i}\right)$ can be calculated using ${ }^{48}$ :

$$
\vec{H}_{r}=\frac{1}{4 \pi} \int_{V} \vec{J}\left(\overrightarrow{r^{\prime}}\right) \times \frac{\left(\vec{r}-\overrightarrow{r^{\prime}}\right)}{\left|\vec{r}-\overrightarrow{r^{\prime}}\right|^{3}} d^{3} \overrightarrow{r^{\prime}}
$$

where $J$ is the current density. The overall magnetic field is averaged at mid-thickness of the layer of interest. 
Received: 25 May 2020; Accepted: 8 December 2020

Published online: 08 January 2021

\section{References}

1. Kim, J., Campbell, A. S., de Ávila, B.E.-F. \& Wang, J. Wearable biosensors for healthcare monitoring. Nat. Biotechnol. 37, 389-406 (2019).

2. Parvez Mahmud, M. A., Huda, N., Farjana, S. H., Asadnia, M. \& Lang, C. Recent advances in nanogenerator-driven self-powered implantable biomedical devices. Adv. Energy Mater. 8, 1701210 (2018).

3. Boto, E. et al. A new generation of magnetoencephalography: room temperature measurements using optically-pumped magnetometers. NeuroImage 149, 404-414 (2017).

4. Fujiwara, K. et al. Magnetocardiography and magnetoencephalography measurements at room temperature using tunnel magnetoresistance sensors. Appl. Phys. Express 11, 023001 (2018).

5. Zuo, S., Heidari, H., Farina, D. \& Nazarpour, K. Miniaturized magnetic sensors for implantable magnetomyography. Adv. Mater. Technol. 5, 2000185 (2020)

6. Jensen, K. et al. Magnetocardiography on an isolated animal heart with a room-temperature optically pumped magnetometer. Sci. Rep. 8, 16218 (2018).

7. Caruso, L. et al. In vivo magnetic recording of neuronal activity. Neuron 95, 1283-1291 (2017).

8. Wang, J., Yang, K., Yang, R., Kong, X. \& Chen, W. Gradiometer module for fetal magnetocardiography detection in a thin magnetically shielded room. In 2018 IEEE International Conference on Applied Superconductivity and Electromagnetic Devices (ASEMD), 1-3 (IEEE, 2018).

9. Kim, Y. J., Savukov, I., Huang, J.-H. \& Nath, P. Magnetic microscopic imaging with an optically pumped magnetometer and flux guides. Appl. Phys. Lett. 110, 043702 (2017).

10. Limes, M. E. et al. Portable magnetometry for detection of biomagnetism in ambient environments. Phys. Rev. Appl. 14, 011002 (2020).

11. Sulai, I. A. et al. Characterizing atomic magnetic gradiometers for fetal magnetocardiography. Rev. Sci. Instrum. 90, 085003 (2019).

12. Liu, K. et al. Microfabricated SERF atomic magnetometers for measurement of weak magnetic field. In 2020 IEEE 70th Electronic Components and Technology Conference (ECTC) 991-996 (2020).

13. Strand, S. et al. Low-cost fetal magnetocardiography: a comparison of superconducting quantum interference device and optically pumped magnetometers. J. Am. Heart Assoc. 8, e013436 (2019).

14. Tierney, T. M. et al. Optically pumped magnetometers: from quantum origins to multi-channel magnetoencephalography. NeuroImage 199, 598-608 (2019).

15. Vemishetty, N. et al. Low power personalized ECG based system design methodology for remote cardiac health monitoring. IEEE Access 4, 8407-8417 (2016).

16. Cardoso, S. et al. Challenges and trends in magnetic sensor integration with microfluidics for biomedical applications. J. Phys. D Appl. Phys. 50, 213001 (2017).

17. Suess, D. et al. Topologically protected vortex structures for low-noise magnetic sensors with high linear range. Nat. Electron. 1, 362-370 (2018).

18. Shafique, M. et al. An overview of next-generation architectures for machine learning: Roadmap, opportunities and challenges in the IoT era. In 2018 Design, Automation \& Test in Europe Conference \& Exhibition (DATE), 827-832 (IEEE, 2018).

19. Kwon, J. H., Kwak, W. Y. \& Cho, B. K. Magnetization manipulation of a flexible magnetic sensor by controlled stress application. Sci. Rep. 8, 15765 (2018).

20. Hirohata, A. et al. Review on spintronics: principles and device applications. J. Magn. Magn. Mater. 509, 166711 (2020).

21. Pannetier-Lecoeur, M. et al. Magnetocardiography with sensors based on giant magnetoresistance. Appl. Phys. Lett. 98, 153705 (2011).

22. Cardoso, S. et al. Magnetic tunnel junction sensors with ptesla sensitivity. Microsyst. Technol. 20, 793-802 (2014).

23. Giouroudi, I. \& Hristoforou, E. Perspective: magnetoresistive sensors for biomedicine. J. Appl. Phys. 124, 030902 (2018).

24. Zheng, C. et al. Magnetoresistive sensor development roadmap (non-recording applications). IEEE Trans. Magn. 55, 1-30 (2019).

25. Adem, S., Jain, S. \& Sveiven, M. Giant magnetoresistive biosensors for real-time quantitative detection of protease activity. Sci. Rep. 10, 1-10 (2020).

26. Valadeiro, J. P. et al. Strategies for ptesla field detection using magnetoresistive sensors with a soft pinned sensing layer. IEEE Trans. Magn. 51, 1-4 (2015).

27. Valadeiro, J. et al. Hybrid integration of magnetoresistive sensors with MEMS as a strategy to detect ultra-low magnetic fields. Micromachines 7, 88 (2016).

28. Paz, E., Serrano-Guisan, S., Ferreira, R. \& Freitas, P. Room temperature direct detection of low frequency magnetic fields in the $100 \mathrm{pt} / \mathrm{hz} 0.5$ range using large arrays of magnetic tunnel junctions. J. Appl. Phys. 115, 17E501 (2014).

29. Weitensfelder, H. et al. Comparison of sensitivity and low-frequency noise contributions in giant-magnetoresistive and tunnelingmagnetoresistive spin-valve sensors with a vortex-state free layer. Phys. Rev. Appl. 10, 054056 (2018).

30. Kim, S., Choi, J. \& Lim, S. Tailoring of magnetic properties of giant magnetoresistance spin valves via insertion of ultrathin nonmagnetic spacers between pinned and pinning layers. Sci. Rep. 19, 1-9 (2019).

31. Silva, M., Leitao, D. C., Cardoso, S. \& Freitas, P. P. Toward ptesla detectivities maintaining minimum sensor footprint with vertical packaging of spin valves. IEEE Trans. Magn. 53, 1-5 (2016)

32. Silva, M., Leitao, D. C., Cardoso, S. \& Freitas, P. MnNi-based spin valve sensors combining high thermal stability, small footprint and pTesla detectivities. AIP Adv. 8, 056644 (2018).

33. Torrejon, J. et al. Multiple giant-magnetoresistance sensors controlled by additive dipolar coupling. Phys. Rev. Appl. 13, 034031 (2020).

34. Camsari, K. Y., Ganguly, S. \& Datta, S. Modular approach to spintronics. Sci. Rep. 5, 10571 (2015).

35. Ripka, P. Review of fluxgate sensors. Sens. Actuators A 33, 129-141 (1992).

36. Harada, S., Sasada, I. \& Hang, F. Development of a one-dimensional fluxgate array and its application to magnetocardiogram measurements. Electron. Commun. Jpn. 98, 20-26 (2015).

37. Song, S. et al. Excitation module for orthogonal fundamental mode fluxgate sensor. J. Instrum. 15, P05007-P05007 (2020).

38. Bartington Instruments. Mag-13 three-axis magnetic field sensors. https://www.bartington.com/mag-13/. Accessed Apr 2020.

39. TDK. Tdk biomagnetic sensor. https://product.tdk.com/info/en/techlibrary/developing/bio-sensor/index.html. Accessed Apr 2020.

40. Wang, M., Wang, Y., Peng, L. \& Ye, C. Measurement of triaxial magnetocardiography using high sensitivity tunnel magnetoresistance sensor. IEEE Sens. J. 19, 9610-9615 (2019).

41. Dieny, B., Gurney, B., Metin, S., Parkin, S. \& Speriosu, V. Magnetoresistive sensor based on the spin valve effect. US Patent 5206590A (1992).

42. Heim, D. E. et al. Design and operation of spin valve sensors. IEEE Trans. Magn. 30, 316-321 (1994).

43. Freitas, P. P., Ferreira, R., Cardoso, S. \& Cardoso, F. Magnetoresistive sensors. J. Phys. Condens. Matter 19, 165221 (2007).

44. Teixeira, J. et al. Ruderman-Kittel-Kasuyama-Yoshida and néel contributions to the interlayer coupling of MNIR-based spin valves: influence of deposition rate, roughness and spacer thickness. J. Appl. Phys. 103, 07F319 (2008). 
45. Silva, A. V. et al. Linearization strategies for high sensitivity magnetoresistive sensors. Eur. Phys. J. Appl. Phys. 72, 10601 (2015).

46. Ro, J.-C., Choi, Y.-S., Suh, S.-J. \& Lee, H.-J. Effect of microstructures on exchange anisotropy in Mn-Ir/Ni-Fe exchange-biased mutilayers with various stacking structures. IEEE Trans. Magn. 35, 3925-3927 (1999).

47. Bi, X., Gan, L., Ma, X., Gong, S. \& Xu, H. Change of coercivity of magnetic thin films with non-magnetic layers and applications to spin valve. J. Magn. Magn. Mater. 268, 321-325 (2004).

48. Bertram, H. N. Theory of Magnetic Recording (Cambridge University Press, Cambridge, 1994).

\title{
Acknowledgements
}

This work has received funding from European Structural \& Investment Funds through the COMPETE Program, from National Funds through the Instituto de Nanociencia e Nanotecnologia (IN) Associated Laboratory, National Infrastructure Roadmap NORTE-01-0145-FEDER-22090, MagScopy4IHC - LISBOA-010145-FEDER-031200, and PTDC/NAN-MAT/31688/2017. FCT funding of the Research Unit INESC MN (UID/053672020) through plurianual BASE and PROGRAMATICO financing. D.C. Leitao acknowledges financial support through FSE/POPH. M. Silva thanks FCT Doctoral Programme AIM Grant PD/BD/128206/2016. F. Franco acknowledges FCT Grant SFRH/BD/111538/2015.

\section{Author contributions}

P.P.F. e S.C. conceived and supervisor the experiment. M.S. deposited, fabricated and characterized the sensors. M.S. and F.F. developed the numerical model. M.S., F.F. and D.C.L. analyzed and interpreted the results and wrote the paper. All authors contributed for the final manuscript.

\section{Competing interests}

The authors declare no competing interests.

\section{Additional information}

Supplementary information is available for this paper at https://doi.org/10.1038/s41598-020-79856-0.

Correspondence and requests for materials should be addressed to M.S.

Reprints and permissions information is available at www.nature.com/reprints.

Publisher's note Springer Nature remains neutral with regard to jurisdictional claims in published maps and institutional affiliations.

\begin{abstract}
(c) (i)
Open Access This article is licensed under a Creative Commons Attribution 4.0 International License, which permits use, sharing, adaptation, distribution and reproduction in any medium or format, as long as you give appropriate credit to the original author(s) and the source, provide a link to the Creative Commons licence, and indicate if changes were made. The images or other third party material in this article are included in the article's Creative Commons licence, unless indicated otherwise in a credit line to the material. If material is not included in the article's Creative Commons licence and your intended use is not permitted by statutory regulation or exceeds the permitted use, you will need to obtain permission directly from the copyright holder. To view a copy of this licence, visit http://creativecommons.org/licenses/by/4.0/.
\end{abstract}

(C) The Author(s) 2021 